\title{
Phytotherapy in reducing glycemic index and testicular oxidative stress resulting from induced diabetes: a review
}

\author{
J. S. Oliveira ${ }^{a}$, A. A. N. Silva ${ }^{b}$ and V. A. Silva Junior ${ }^{*}$ \\ aPrograma de Pós-graduação em Ciência Animal Tropical, Universidade Federal Rural de Pernambuco - UFRPE, \\ Rua Dom Manuel de Medeiros, Dois Irmãos, CEP 52171-900, Recife, PE, Brazil \\ bPrograma de Pós-graduação em Biociência Animal, Universidade Federal Rural de Pernambuco - UFRPE, \\ Rua Dom Manuel de Medeiros, Dois Irmãos, CEP 52171-900, Recife, PE, Brazil \\ 'Departamento de Medicina Veterinária, Universidade Federal Rural de Pernambuco - UFRPE, \\ Rua Dom Manuel de Medeiros, Dois Irmãos, CEP 52171-900, Recife, PE, Brazil \\ *e-mail: valdemiroamaro@gmail.com
}

Received: June 30, 2015 - Accepted: November 18, 2015 - Distributed: February 28, 2017

(With 1 figure)

\begin{abstract}
Oxidative stress plays a main role in the development of diabetes complications. The impairment of gonadal antioxidant potential and endocrine disturbance in diabetic males causes testicular damage and failure in sperm production. Plants have been widely used to control diabetes due their hypoglycemic and antioxidant potential, contributing towards the recovery of testicular function. Current study comprises a review of the literature on the main medicinal plants used in the recovery of testicular oxidative damage in animals with experimental diabetes. Eighteen plant species in the nineteen studies selected from the search strategy were evaluated. Plant extracts were evaluated according to their effects on blood glucose and insulin levels, antioxidant enzymes and oxidant levels, lipid peroxidation, total protein, testosterone levels, gonadosomatic index, diameter of seminiferous tubules, seminiferous epithelium height and integrity, number of germ cells at stage VII and apoptosis in the seminiferous epithelium, sperm production, motility, viability and morphology. After the analysis of the studies, it was observed that plant species, used alone or in combination, may control testicular oxidative damage triggered by diabetes. The antioxidant potential varies among species, with some plants proving to have a better performance in the recovery of reproduction parameters than others.
\end{abstract}

Keywords: testis, medicinal plants, antioxidants, spermatogenesis.

\section{Fitoterapia na redução da glicemia e do estresse oxidativo testicular resultantes do diabetes experimental: uma revisão}

\begin{abstract}
Resumo
O estresse oxidativo desempenha um dos principais papéis no desenvolvimento das complicações do diabetes. Em machos diabéticos, as alterações ao potencial antioxidante gonadal e os distúrbios endócrinos resultam em danos testiculares e falhas na produção de espermatozoides. Plantas têm sido amplamente utilizadas como um método de controle do diabetes devido aos seus potenciais hipoglicemiante e antioxidante, contribuindo para a recuperação da função testicular. Assim, o objetivo deste estudo é fazer uma revisão da literatura sobre as principais plantas medicinais utilizadas na recuperação de danos oxidativos testiculares em animais induzidos ao diabetes experimental. Nos dezenove estudos selecionados a partir da estratégia de busca, dezoito espécies de plantas foram utilizadas. Os extratos das espécies vegetais foram avaliados de acordo com os seus efeitos nas concentrações de glicose e insulina, níveis das enzimas antioxidantes e dos oxidantes, peroxidação lipídica, quantidade de proteínas totais, níveis de testosterona, índice gonadossomático, diâmetro dos túbulos seminíferos, altura e integridade do epitélio seminífero, número de células germinativas no estágio VII, número de células em apoptose no epitélio seminífero, produção, motilidade, viabilidade e morfologia espermática. Depois das análises aos estudos, foi observado que as espécies vegetais, sozinhas ou em combinação, podem ajudar a controlar os danos oxidativos testiculares desencadeados pelo diabetes. Contudo, o potencial antioxidante varia entre as espécies, algumas tenho uma melhor performance na recuperação dos parâmetros reprodutivos do que outras.
\end{abstract}

Palavras-chave: testículo, plantas medicinais, antioxidantes, espermatogênese. 


\section{Introduction}

Diabetes is a metabolic disease characterized by increased blood glucose levels due to failure in insulin production or function. The persistence of high levels of blood glucose compromises homeostasis and promotes body damage, including retinopathy, nephropathy, cardiovascular complications and sexual dysfunction (Van Belle et al., 2011).

Oxidative stress is the main cause of diabetes complications (Maritim et al., 2003). It mainly originates from high blood glucose levels, where the reactive oxygen species (ROS) and the free radicals (FR) production exceeds the defense capacity of the organism and disrupt the cellular reduction-oxidation balance (Dowling and Simmons, 2009; Cansian et al., 2015; Takao et al., 2015).

The impairment of gonadal antioxidant potential of the male reproduction system causes testicular disturbance and failure in sperm production (Mallick et al., 2007; Hamden et al., 2008). Furthermore, disorders in the endocrine control of spermatogenesis have also been observed in diabetic patients, with reduced levels of essential hormones, such as FSH, LH and testosterone (Ballester et al., 2004; Agbaje et al., 2007).

The body's natural antioxidants act by neutralizing ROS or by preventing the release of ions responsible for initiating lipid peroxidation (Shah and Khan, 2014). Superoxide dismutase (SOD) and catalase (CAT) are key enzymes that act against toxicity by controlling ROS levels (Singh et al., 2013). It has been reported that SOD and CAT levels are reduced in diabetic patients, which, along with increased Bcl-2 and Bax protein, lead to apoptosis of the testicular germ cells (Chatterjee et al., 2012).

Thus, glycemic control and increased antioxidant protection are necessary to reduce the body damage in diabetic conditions (Singh et al., 2013). The most common way to achieve nearly-normal glucose levels is the daily administration of insulin or insulin pumps (Ratner et al., 2000). Although other methods such as the use of sulfonylureas and biguanides may also be applied, they are expensive and may cause complications involving toxicity, gastrointestinal disorders and weight gain (Modak et al., 2007).

Therefore, plants have been widely used as a method for the control of diabetes due to their products in secondary metabolism and their hypoglycemic and antioxidant potential (Singh et al., 2013; Ghosh and Roy, 2013). These products minimize the damage caused by the accumulation of free radicals and contribute towards the recovery of testicular functions (Modak et al., 2007; Shah and Khan, 2014). Thus, the aim of current literature review is to report on the main medicinal plants with antioxidant and hypoglycemic potential used in the recovery of testicular oxidative damage in animals with experimental diabetes.

\section{Material and Methods}

Articles were identified by searching the MedLine, PubMed and Scopus databases in October 2014. There was no article restriction related to the year of publication.
In the search strategy, the following combinations of words were used: 1. plants AND oxidative stress AND diabetes AND testes AND rat, 2. plants AND oxidative stress AND diabetes AND sperm AND rat, 3. plants AND oxidative stress AND diabetes AND spermatogenesis AND rat, 4. plants AND antioxidant AND diabetes AND testes AND rat, 5. plants AND antioxidant AND diabetes AND spermatogenesis AND rat, 6. plants AND antioxidant AND diabetes AND sperm AND rat.

The selected studies were analyzed and organized according to the year of publication, drugs used to induce diabetes, plant species used for treatment, treatment duration, testicular and reproductive parameters evaluated and treatment efficiency.

\section{Results and Discussion}

The literature comprised 429 articles. After the removal of duplicates and after analysis in accordance with the study purposes, 19 articles were selected. Search strategy and the selection of items are summarized in Figure 1.

Table 1 shows the features of the selected studies according to authors, year of publication, drug used to induce experimental type 1 diabetes, plant species used in the extract, type and duration of treatment and testicular parameters used for assessing the treatments' efficiency.

The selected papers registered 18 plant species used: Ajuga iva (southern bugle), Anacardium occidentale (cashew), Cassia sophera, Chlorophytum borivilianum (safed musli), Cnidoscolus aconitifolius, Coccinia indica, Danae racemosa (poet's laurel), Dracaena arborea, Eugenia jambolana (jambul), Hyphaene thebaica (doum palm), Momordica charantia (karela), Morus alba (white mulberry), Mucuna pruriens (velvet bean), Musa paradisiaca (banana), Phoenix dactylifera (date palm), Sida cordata, Tamarindus indica (tamarind) and Urtica dioica (stinging nettle). Musa paradisiaca was the most frequent species and its root extract was present in $21 \%$ of articles. Leaves of Coccinia indica and seeds of Eugenia jambolana were reported in $15.8 \%$ of studies whilst seeds of Tamarindus indica were used in $10.5 \%$. The other species were not repeated in the methods.

Plant extract had its antioxidant potential assessed in only one diabetic experimental group in $38 \%$ of the studies, whereas in $14.2 \%$ of the studies, the treatment had been provided to a group of healthy animals, which were used as a control group. The above methodology is important due to the possibility of identifying toxic effects in the treatment by the plant species. Extracts were also used in different doses $(14.2 \%)$ and with different chemical compositions $(14.2 \%)$, to determine the most effective treatment. Moreover, the protective role of the extract was evaluated in $9.4 \%$ of studies; in other words, the treatment started before the induction of experimental diabetes.

Blood glucose level during the experiment or on euthanasia day was assessed in $52.6 \%$ of the studies. The authors with the greatest success on hypoglycemic potential of plant species were Mallick et al. (2007), Chatterjee et al. (2012) 


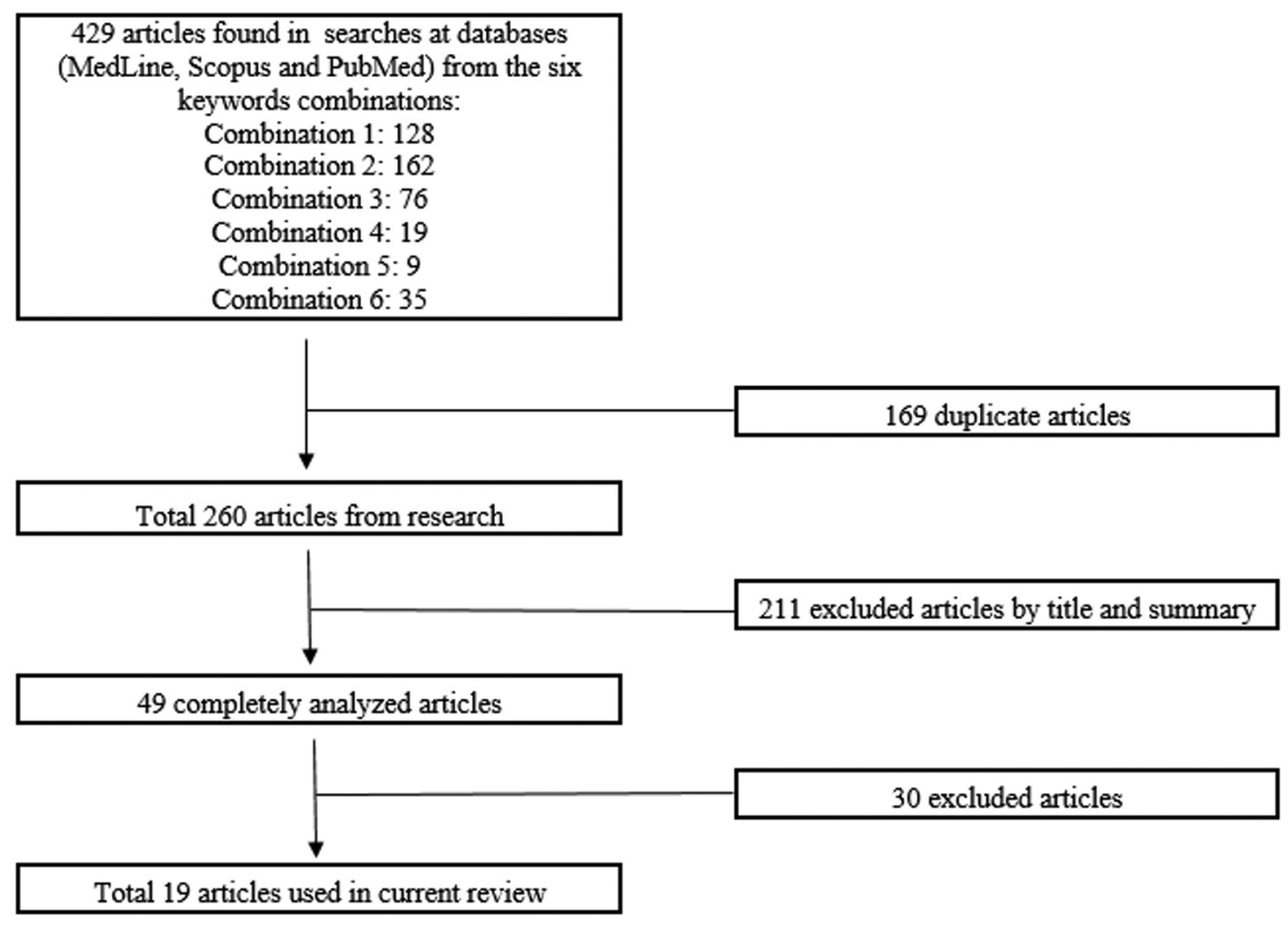

Figure 1. Search strategy in MedLine, Scopus and PubMed databases and selection of articles on the use of plants for the recovery of testicular oxidative damage resulting from experimental type I diabetes.

and Shah and Khan (2014), who restored glucose levels to those in the control group (see Table 2).

It has been generally observed that increase in the treatment period results in a better response and, consequently, a greater reduction in blood glucose levels. However, Shah and Khan (2014) had better results with higher concentrations in the plant extract. Whereas Chlorophytum borivilianum, Morus alba and Urtica dioica had low efficiency in the reduction of blood glucose levels, Dracaena arborea had no effect and the treated animals revealed similar rates as those for untreated diabetics, regardless of extract concentration.

In $31.6 \%$ of studies that evaluated the blood insulin concentration, Mallick et al. (2010) obtained the most efficient results, restoring the low levels observed in diabetic animals to those of control group. However, Mallick et al. (2007), Chatterjee et al. (2012, 2013), Hajizadeh et al. (2014) and Shah and Khan (2014) reported a recovery compared to untreated diabetic animals. According to Chatterjee et al. (2013), the extract used for treatment of diabetic animals increased the production of the transforming growth factor $\beta 1$ (TGF- $\beta 1$ ) which prevents the infiltration of lymphocytes in pancreatic islet and protects $\beta$-cells while providing a safe environment for cellular regeneration.

Gonadosomatic index (GSI), the ratio between testicular weights and body weight, was measured in only $21 \%$ of the studies. All studies revealed that rates for untreated diabetic animals were significantly lower than those of the other experimental groups and the treatment with plant species resulted in recovery. In spite of the absence of testicular weight in such studies, the reduction of GSI, or rather, the percentage of body weight allocated in the gonads, indicated a decrease in testis size. Specifically in Chatterjee et al. (2013), in which body weight of experimental groups had no significant difference, the reduction of such index features drastic testicular damage. Wankeu-Nya et al. (2013) also observed a testicular atrophy in diabetes-induced animals, partially recovered by treatment.

According to França and Russell (1998), testicular weight is an important assessor of androgenic activity and sperm production in mammals. Chatterjee et al. (2013) and Ghosh et al. (2014) reported that decrease in GSI and, consequently, in testis weight, is the result of diabetic low testosterone and insulin levels.

Levels of antioxidant enzymes, including catalase, glutathione reductase, glutathione s-transferase, glutathione peroxidase, peroxidase and superoxide dismutase, were investigated in $63 \%$ of studies. All papers reported reduced levels of antioxidant enzymes in untreated diabetic animals. However, only $10.4 \%$ of the studies measured oxidant levels, such as nitric oxide, nitrite/nitrate and hydrogen peroxide. Giribabu et al. (2014) and Shah and Khan (2014) observed increased levels of these oxidants respectively 
Table 1. Methodological characteristics of selected studies on the use of plants as therapy against testicular oxidative effect of experimental Type 1 diabetes.

\begin{tabular}{|c|c|c|c|c|}
\hline Authors & $\begin{array}{l}\text { Drug used } \\
\text { to induce } \\
\text { diabetes }\end{array}$ & $\begin{array}{l}\text { Plant species } \\
\text { used for } \\
\text { treatment }\end{array}$ & Treatment & $\begin{array}{c}\text { Testicular parameters } \\
\text { analyzed }\end{array}$ \\
\hline $\begin{array}{l}\text { Raza et al. } \\
\quad(2004)\end{array}$ & Streptozotocin & $\begin{array}{l}\text { Momordica } \\
\text { charantia }\end{array}$ & $\begin{array}{l}\text { One healthy and one diabetic } \\
\text { experimental } \\
\text { group received orally the extract } \\
\text { of seeds of } \\
\text { Momordica charantia at the } \\
\text { dose of } \\
10 \mathrm{ml} / \mathrm{kg} \text { daily for } 14 \text { days }\end{array}$ & $\begin{array}{l}\text { Endogenous peroxidase } \\
\text { activity, localization of GST } \\
\text { alpha }\end{array}$ \\
\hline $\begin{array}{l}\text { Mallick et al. } \\
\qquad(2007)\end{array}$ & Streptozotocin & $\begin{array}{l}\text { Musa } \\
\text { paradisiaca, } \\
\text { Tamarindus } \\
\text { indica, } \\
\text { Eugenia } \\
\text { jambolana } \\
\text { and Coccinia } \\
\text { indica }\end{array}$ & $\begin{array}{l}\text { One diabetic experimental } \\
\text { group was orally treated with a } \\
\text { compound of specific parts of } \\
\text { the species Musa paradisiaca, } \\
\text { Tamarindus indica, Eugenia } \\
\text { jambolana Coccinia indica at } \\
\text { the dose of } 60 \mathrm{mg} / 0.5 \mathrm{ml} \text { olive } \\
\text { oil } / 100 \mathrm{~g} \text { body weight } / \text { twice } \\
\text { daily for } 14 \text { days }\end{array}$ & $\begin{array}{l}\text { Gonadosomatic index, serum } \\
\text { testosterone, sperm count and } \\
\text { viability, testicular glucose } \\
\text { level, activities of testicular } \\
\text { catalase and glutathione } \\
\text { peroxidase, lipid peroxidation, } \\
\text { quantification of germ cells } \\
\text { at stage VII in seminiferous } \\
\text { epithelial cycle, seminiferous } \\
\text { tubules diameter, Leydig cell } \\
\text { nuclear area }\end{array}$ \\
\hline
\end{tabular}

One diabetic experimental group received orally the aqueous extract of Ajuga iva at the dose

Hamden et al. (2008)

Alloxan Ajuga iva

Azeez et al. (2010) Alloxan

Cnidoscolus aconitifolius

of $50 \mathrm{mg} / \mathrm{kg}$ for three weeks.

Another experimental group received the same extract in the same concentration for three weeks before diabetes induction

Three diabetics experimental groups received orally doses of ethanol extract of Cnidoscolus aconitifolius in different concentrations $(100 \mathrm{mg} / \mathrm{kg}$, $500 \mathrm{mg} / \mathrm{kg}$ and $1000 \mathrm{mg} / \mathrm{kg}$ ) daily for 28 days Musa fraction of hydro-methanolic

Mallick et al. (2010)

Streptozotocin paradisiaca and Coccinia indica
Sperm count and motility,

$17-\beta$ estradiol levels, serum testosterone, lipid peroxidation, antioxidant enzymes activity, total protein
Motility, number, morphology and viability of sperm
One diabetic experimental group was orally treated with hexane extract of root of Musa

paradisiaca and leaf of Coccinia indica at the dose of $2 \mathrm{mg} / 0.2 \mathrm{ml}$ olive oil/100 $\mathrm{g}$ body weight for 45 days
Serum testosterone, sperm count and viability, antioxidant enzymes activity, lipid peroxidation, incidence of apoptosis in seminiferous tubules, quantification of germ cells at stage VII in seminiferous epithelial cycle and giant cell in testicular section

One healthy and one diabetic experimental group were orally treated with the extract of Serum testosterone
Shahreari et al. Streptozotocin
$(2010)$ $\begin{aligned} & \text { Danae } \\ & \text { racemosa }\end{aligned}$
Danae racemosa at the dose of $400 \mathrm{mg} / \mathrm{kg}$ daily for four weeks 
Table 1. Continued...

\begin{tabular}{|c|c|c|c|c|}
\hline Authors & $\begin{array}{c}\text { Drug used } \\
\text { to induce } \\
\text { diabetes }\end{array}$ & $\begin{array}{c}\text { Plant species } \\
\text { used for } \\
\text { treatment }\end{array}$ & Treatment & $\begin{array}{c}\text { Testicular parameters } \\
\text { analyzed }\end{array}$ \\
\hline $\begin{array}{l}\text { Golalipour et al. } \\
\qquad(2011)\end{array}$ & Streptozotocin & Urtica dioica & $\begin{array}{l}\text { One experimental group received } \\
\text { an intraperitoneal injection with } \\
\text { extract of Urtica dioica leaves at } \\
\text { a dose of } 100 \mathrm{mg} / \mathrm{kg} \text { for } 5 \text { days. } \\
\text { On the sixth day, the animals } \\
\text { were induced to experimental } \\
\text { diabetes }\end{array}$ & $\begin{array}{l}\text { Seminiferous tubules diameter, } \\
\text { seminiferous epithelial height }\end{array}$ \\
\hline $\begin{array}{l}\text { Chatterjee et al. } \\
\qquad(2012)\end{array}$ & Streptozotocin & $\begin{array}{l}\text { Musa } \\
\text { paradisiaca }\end{array}$ & $\begin{array}{l}\text { One diabetic experimental } \\
\text { group was orally treated } \\
\text { with ethyl acetate fraction of } \\
\text { hydromethanolic extract of } \\
\text { root of Musa paradisiaca at the } \\
\text { dose of } 20 \mathrm{mg} / 0.5 \mathrm{ml} \text { distilled } \\
\text { water } / 100 \mathrm{~g} \text { body weight }\end{array}$ & $\begin{array}{l}\text { Antioxidant enzymes activity, } \\
\text { lipid peroxidation, testicular } \\
\text { cholesterol level, sperm count } \\
\text { and motility, testicular Bax- } \alpha \\
\text { mRNA expression pattern, } \\
\text { seminiferous tubules diameter } \\
\text { and testicular histology }\end{array}$ \\
\hline $\begin{array}{l}\text { Suresh et al. } \\
\qquad(2013)\end{array}$ & Streptozotocin & $\begin{array}{l}\text { Mucuna } \\
\text { pruriens }\end{array}$ & $\begin{array}{l}\text { One healthy and one diabetic } \\
\text { experimental group received } \\
\text { orally the ethanolic extract of } \\
\text { seeds of Mucuna pruriens at the } \\
\text { dose of } 200 \mathrm{mg} / \mathrm{kg} \text { once a day } \\
\text { for } 60 \text { days }\end{array}$ & $\begin{array}{l}\text { Sperm count, morphology, } \\
\text { motility, DNA integrity, } \\
\text { quantitative assessment of } \\
\text { DNA damage, estimation } \\
\text { of lipid peroxidation and } \\
\text { antioxidant }\end{array}$ \\
\hline $\begin{array}{l}\text { Chatterjee et al. } \\
\qquad(2013)\end{array}$ & Streptozotocin & $\begin{array}{l}\text { Musa } \\
\text { paradisiaca, } \\
\text { Tamarindus } \\
\text { indica, } \\
\text { Eugenia } \\
\text { jambolana } \\
\text { and Coccinia } \\
\text { indica }\end{array}$ & $\begin{array}{l}\text { One diabetic experimental } \\
\text { group was orally treated with } \\
\text { a compound of specific parts } \\
\text { of solvent fractions of } M u s a \\
\text { paradisiaca, Tamarindus } \\
\text { indica, Eugenia jambolana and } \\
\text { Coccinia indica at the dose of } \\
10 \mathrm{mg} / 0.5 \mathrm{ml} \text { of } 2 \% \text { Tween } \\
80 / 100 \mathrm{~g} \text { body weight twice daily } \\
\text { for } 28 \text { days }\end{array}$ & $\begin{array}{l}\text { Gonadosomatic index, } \\
\text { sperm count and motility, } \\
\text { seminal vesicular fructose } \\
\text { and testicular cholesterol } \\
\text { levels, serum testosterone, } \\
\text { antioxidant enzymes } \\
\text { activity, estimation of lipid } \\
\text { peroxidation, Bax- } \alpha \text { protein } \\
\text { expression, histological } \\
\text { evaluation of the testis }\end{array}$ \\
\hline $\begin{array}{l}\text { Michael et al. } \\
\text { (2013) }\end{array}$ & Alloxan & $\begin{array}{l}\text { Phoenix } \\
\text { dactylifera }\end{array}$ & $\begin{array}{l}\text { Two diabetic experimental } \\
\text { groups were orally treated with } \\
\text { solutions of the component } \\
1 \text { (diosmetin } 7-\mathrm{O}-\beta \text {-L- } \\
\text { arabinofuranosyl }(1 \rightarrow 2) \\
\beta \text {-D-apiofuranoside) and } \\
\text { component } 2 \text { (diosmetin } 7-\mathrm{O}-\beta \text { - } \\
\text { D-apiofuranoside) isolated from } \\
\text { the acetone extract of Phoenix } \\
\text { dactylifera }\end{array}$ & Serum testosterone \\
\hline $\begin{array}{l}\text { Salib et al. } \\
\text { (2013) }\end{array}$ & Alloxan & $\begin{array}{l}\text { Hyphaene } \\
\text { thebaica }\end{array}$ & $\begin{array}{l}\text { One diabetic experimental group } \\
\text { received orally an aqueous } \\
\text { solution of the soluble fraction } \\
\text { of Hyphaene thebaica ( } 20 \mathrm{mg} / \\
\mathrm{kg} \text { ) daily for } 30 \text { days. A second } \\
\text { diabetic group received orally a } \\
\text { component } 5 \text { solution of soluble } \\
\text { aqueous fraction of Hyphaene } \\
\text { thebaica }(20 \mathrm{mg} / \mathrm{kg}) \text { daily for } \\
30 \text { days }\end{array}$ & Serum testosterone \\
\hline
\end{tabular}


Table 1. Continued...

\begin{tabular}{|c|c|c|c|c|}
\hline Authors & $\begin{array}{c}\text { Drug used } \\
\text { to induce } \\
\text { diabetes }\end{array}$ & $\begin{array}{l}\text { Plant species } \\
\text { used for } \\
\text { treatment }\end{array}$ & Treatment & $\begin{array}{c}\text { Testicular parameters } \\
\text { analyzed }\end{array}$ \\
\hline $\begin{array}{l}\text { Singh et al. } \\
\text { (2013) }\end{array}$ & Streptozotocin & $\begin{array}{l}\text { Cassia } \\
\text { sophera }\end{array}$ & $\begin{array}{l}\text { One diabetic experimental } \\
\text { group was orally treated with } \\
\text { the aqueous extract of Cassia } \\
\text { sophera }(200 \mathrm{mg} / \mathrm{kg}) \text { for } 28 \text { days }\end{array}$ & $\begin{array}{l}\text { Lipid peroxidation, } \\
\text { concentration of enzymatic } \\
\text { and non-enzymatic } \\
\text { antioxidants and protein } \\
\text { concentration }\end{array}$ \\
\hline $\begin{array}{l}\text { Ukwenya et al. } \\
\text { (2013) }\end{array}$ & Streptozotocin & $\begin{array}{l}\text { Anacardium } \\
\text { occidentale }\end{array}$ & $\begin{array}{l}\text { One diabetic experimental group } \\
\text { received orally } 300 \mathrm{mg} / \mathrm{kg} \text { of the } \\
\text { methanol extract of Anacardium } \\
\text { occidentale sheet daily for } 16 \\
\text { days }\end{array}$ & $\begin{array}{l}\text { Lipid peroxidation, } \\
\text { antioxidant enzymes activity, } \\
\text { histological evaluation of the } \\
\text { testis }\end{array}$ \\
\hline $\begin{array}{l}\text { Wankeu-Nya et al. } \\
\qquad(2013)\end{array}$ & Streptozotocin & $\begin{array}{l}\text { Dracaena } \\
\text { arborea }\end{array}$ & $\begin{array}{l}\text { Two diabetic experimental group } \\
\text { were orally treated with the } \\
\text { aqueous extract and the ethanol } \\
\text { extract of Dracaena arborea } \\
\text { at a dose of } 100 \mathrm{mg} / \mathrm{kg} \text { and } \\
500 \mathrm{mg} / \mathrm{kg} \text { daily for } 3 \text { weeks }\end{array}$ & Testicular morphology \\
\hline $\begin{array}{l}\text { Ghosh et al. } \\
\text { (2014) }\end{array}$ & Streptozotocin & $\begin{array}{l}\text { Eugenia } \\
\text { jambolana }\end{array}$ & $\begin{array}{l}\text { One diabetic experimental } \\
\text { group was orally treated with } \\
\text { the ethyl acetate fraction of } \\
\text { the hydromethanolic extract of } \\
\text { Eugenia jambolana at a dose } \\
\text { of } 20 \mathrm{mg} / 0.5 \mathrm{ml} \text { of distilled } \\
\text { water } / 100 \mathrm{~g} \text { body weight for } \\
60 \text { days }\end{array}$ & $\begin{array}{l}\text { Gonadosomatic index, } \\
\text { seminiferous tubules diameter, } \\
\text { sperm count, seminal vesicular } \\
\text { fructose level, quantification } \\
\text { of germ cells at stage VII in } \\
\text { seminiferous epithelial cycle, } \\
\text { antioxidant enzymes activity, } \\
\text { serum testosterone, analysis } \\
\text { of Bax and Bcl-2 gene } \\
\text { expression }\end{array}$ \\
\hline $\begin{array}{l}\text { Giribabu et al. } \\
\text { (2014) }\end{array}$ & Streptozotocin & $\begin{array}{l}\text { Chlorophytum } \\
\text { borivilianum }\end{array}$ & $\begin{array}{l}\text { Two experimental diabetic group } \\
\text { orally treated with aqueous } \\
\text { Chlorophytum borivilianum } \\
\text { extract at a dose of } 250 \mathrm{mg} / \mathrm{kg} \\
\text { and } 500 \mathrm{mg} / \mathrm{kg} \text { for } 28 \text { days }\end{array}$ & $\begin{array}{l}\text { Sperm count, morphology, } \\
\text { motility and viability, sperm's } \\
\text { flagella membrane integrity, } \\
\text { lipid peroxidation, hydrogen } \\
\text { peroxide and nitric oxide } \\
\text { levels, sperm total antioxidant } \\
\text { capacity, activity levels of } \\
\text { endogenous antioxidant } \\
\text { enzymes, expression of sperm } \\
\text { caspase-3 }\end{array}$ \\
\hline $\begin{array}{l}\text { Hajizadeh et al. } \\
\text { (2014) }\end{array}$ & Streptozotocin & Morus alba & $\begin{array}{l}\text { One diabetic experimental group } \\
\text { received orally Morus alba } \\
\text { extract at a dose of } 1 \mathrm{~g} / \mathrm{kg} \text { per } \\
\text { day for } 8 \text { weeks }\end{array}$ & $\begin{array}{l}\text { Lipid peroxidation, } \\
\text { antioxidant enzymes activity, } \\
\text { mRNA expression level of } \\
\text { StAR and P450scc }\end{array}$ \\
\hline $\begin{array}{l}\text { Shah and Khan } \\
\text { (2014) }\end{array}$ & Alloxan & Sida cordata & $\begin{array}{l}\text { Two experimental diabetic } \\
\text { groups received orally the ethyl } \\
\text { acetate fraction of Sida cordata } \\
\text { at a dose of } 150 \mathrm{mg} / \mathrm{kg} \text { and } \\
300 \mathrm{mg} / \mathrm{kg} \text { for } 15 \text { days }\end{array}$ & $\begin{array}{l}\text { Lipid peroxidation, hydrogen } \\
\text { peroxide, nitrite and protein } \\
\text { levels }\end{array}$ \\
\hline
\end{tabular}

in sperm and testicular evaluation of untreated diabetic animals. This occurs due to auto-oxidation of glucose and non-enzymatic glycation during hyperglycemia, with the subsequent increase in the production of hydrogen peroxide and superoxide radical (Chatterjee et al., 2013).

Giribabu et al. (2014) noted a reduction in the levels of these oxidative stress indicators in the sperm of diabetic rats and underscored the extract's capacity to eliminate free radicals. More efficient results were obtained when higher extract concentration for ROS levels were administered, also corroborated by Shah and Khan (2014). Thereby, the plants' active principles play an important role in reducing blood glucose levels and in recovering testicular antioxidant defense (Hamden et al., 2008). 
Table 2. Glucose levels after induction of experimental Type 1 diabetes and under treatment with the plant species in the selected studies.

\begin{tabular}{|c|c|c|c|c|c|c|}
\hline \multirow{2}{*}{ Authors } & \multirow{2}{*}{ Species } & \multirow{2}{*}{$\begin{array}{l}\text { Days after } \\
\text { diabetes } \\
\text { induction }\end{array}$} & \multicolumn{4}{|c|}{ Blood glucose level (mg/dL) } \\
\hline & & & $\begin{array}{c}\text { Control } \\
\text { Group }\end{array}$ & $\begin{array}{c}\text { Diabetic } \\
\text { Group }\end{array}$ & Treated Dia & betic Group \\
\hline \multirow{3}{*}{$\begin{array}{l}\text { Mallick et al. } \\
\text { (2007) }\end{array}$} & \multirow{3}{*}{$\begin{array}{l}\text { Musa } \\
\text { paradisiaca, } \\
\text { Tamarindus } \\
\text { indica, } \\
\text { Eugenia } \\
\text { jambolana } \\
\text { and Coccinia } \\
\text { indica }\end{array}$} & 1 & $\cong 70 \mathrm{a}$ & $\cong 310 \mathrm{~b}$ & \multicolumn{2}{|c|}{$\cong 310 \mathrm{~b}$} \\
\hline & & 7 & $\cong 70 \mathrm{a}$ & $\cong 300 \mathrm{~b}$ & \multicolumn{2}{|c|}{$\cong 250 \mathrm{c}$} \\
\hline & & 16 & $\cong 70 \mathrm{a}$ & $\cong 310 \mathrm{~b}$ & \multicolumn{2}{|c|}{$\cong 80 \mathrm{c}$} \\
\hline \multirow{3}{*}{$\begin{array}{l}\text { Mallick et al. } \\
\qquad(2010)\end{array}$} & \multirow{3}{*}{$\begin{array}{l}\text { Musa } \\
\text { paradisiaca } \\
\text { and } \\
\text { Coccinia } \\
\text { indica }\end{array}$} & 1 & $74.3 \pm 5.6 \mathrm{a}$ & $322.3 \pm 5.4 b$ & \multicolumn{2}{|c|}{$329.7 \pm 5.1 b$} \\
\hline & & 15 & $72.6 \pm 5.1 \mathrm{a}$ & $337.2 \pm 5.2 b$ & \multicolumn{2}{|c|}{$163.0 \pm 5.4 \mathrm{c}$} \\
\hline & & 45 & $73.5 \pm 4.9 \mathrm{a}$ & $345.5 \pm 5.3 b$ & \multicolumn{2}{|c|}{$74.41 \pm 5.1 \mathrm{a}$} \\
\hline $\begin{array}{l}\text { Golalipour et al. } \\
\text { (2011) }\end{array}$ & Urtica dioica & 35 & $88.5 \pm 3.3 \mathrm{a}$ & $475.2 \pm 39.6 b$ & \multicolumn{2}{|c|}{$301.7 \pm 80.1 \mathrm{c}$} \\
\hline \multirow{3}{*}{$\begin{array}{l}\text { Chatterjee et al. } \\
\text { (2012) }\end{array}$} & \multirow{3}{*}{$\begin{array}{l}\text { Musa } \\
\text { paradisiaca }\end{array}$} & 1 & $65 \pm 2.9 \mathrm{a}$ & $259 \pm 11.49 b$ & \multicolumn{2}{|c|}{$233 \pm 10.4 \mathrm{c}$} \\
\hline & & 16 & $63 \pm 2.8 \mathrm{a}$ & $259 \pm 11.7 \mathrm{~b}$ & \multicolumn{2}{|c|}{$183 \pm 4.1 \mathrm{~d}$} \\
\hline & & 35 & $63 \pm 2.8 \mathrm{a}$ & $263 \pm 11.76 b$ & \multicolumn{2}{|c|}{$68 \pm 3.1 \mathrm{a}$} \\
\hline \multirow{3}{*}{$\begin{array}{c}\text { Chatterjee et al. } \\
\qquad(2013)\end{array}$} & \multirow{3}{*}{$\begin{array}{l}\text { Musa } \\
\text { paradisiaca, } \\
\text { Tamarindus } \\
\text { indica, } \\
\text { Eugenia } \\
\text { jambolana } \\
\text { and Coccinia } \\
\text { indica }\end{array}$} & 1 & $64.5 \pm 2.9 a$ & $326.7 \pm 14.6 b$ & \multicolumn{2}{|c|}{$343.4 \pm 15.3 b$} \\
\hline & & 7 & $64.5 \pm 2.9 a$ & $356.4 \pm 15.8 b$ & \multicolumn{2}{|c|}{$358.4 \pm 16.0 \mathrm{~b}$} \\
\hline & & 35 & $62.5 \pm 2.7 \mathrm{a}$ & $354.4 \pm 15.7 b$ & \multicolumn{2}{|c|}{$87.4 \pm 4.9 c$} \\
\hline \multirow{3}{*}{$\begin{array}{c}\text { Ukwenya et al. } \\
\text { (2013) }\end{array}$} & \multirow{3}{*}{$\begin{array}{l}\text { Anacardium } \\
\text { occidentale }\end{array}$} & 2 & $\cong 90$ & $\cong 360$ & \multicolumn{2}{|c|}{$\cong 330$} \\
\hline & & 10 & $\cong 100$ & $\cong 370$ & \multicolumn{2}{|c|}{$\cong 180$} \\
\hline & & 16 & $\cong 90$ & $\cong 410$ & \multicolumn{2}{|c|}{$\cong 120$} \\
\hline \multirow{3}{*}{$\begin{array}{c}\text { Wankeu-Nya et al. } \\
\qquad(2013)\end{array}$} & \multirow{3}{*}{$\begin{array}{l}\text { Dracaena } \\
\text { arborea }\end{array}$} & & & & $100 \mathrm{mg} / \mathrm{kg}$ & $500 \mathrm{mg} / \mathrm{kg}$ \\
\hline & & 1 & $98.4 \pm 2.4 \mathrm{a}$ & $374.2 \pm 31.8 b$ & $366.6 \pm 8.7 b$ & $367.8 \pm 21.7 b$ \\
\hline & & 21 & $99.2 \pm 1.0 \mathrm{a}$ & $>600 \mathrm{c}$ & $436.4 \pm 25.9 \mathrm{~d}$ & $424.4 \pm 26.7 \mathrm{~d}$ \\
\hline \multirow{2}{*}{$\begin{array}{l}\text { Giribabu et al. } \\
\text { (2014) }\end{array}$} & Chlorophytum & & & & $250 \mathrm{mg} / \mathrm{kg}$ & $500 \mathrm{mg} / \mathrm{kg}$ \\
\hline & borivilianum & 28 & $93.15 \pm 6.8 \mathrm{a}$ & $424.16 \pm 7.23 b$ & $235.24 \pm 6.78 \mathrm{c}$ & $216.73 \pm 4.84 c$ \\
\hline $\begin{array}{l}\text { Hajizadeh et al. } \\
\text { (2014) }\end{array}$ & Morus alba & 56 & $110.3 \pm 2.5 \mathrm{a}$ & $437.2 \pm 10.2 b$ & 322.4 & $25.6 \mathrm{c}$ \\
\hline Shah and Khan & & & & & $150 \mathrm{mg} / \mathrm{kg}$ & $300 \mathrm{mg} / \mathrm{kg}$ \\
\hline$(2014)$ & Sida cordata & 15 & $83.6 \pm 4.0 \mathrm{a}$ & $241.6 \pm 7.6 b$ & $129.3 \pm 5.3 c$ & $95.6 \pm 3.5^{\mathrm{a}}$ \\
\hline
\end{tabular}

Different letters in the same study represent a significant difference $(\mathrm{p}<0.05)$, their absence indicates lack of statistical information.

Oxidative stress is a main feature in diabetic conditions and results from persistent high blood glucose levels. There is a reduction in the levels of critical enzymes for antioxidant protection under these conditions, leading to increased formation of reactive oxygen species and, consequently, to tissue damage, such as lipid peroxidation and increased rate of apoptosis (Maritim et al., 2003; Ghosh, et al., 2014). Superoxide dismutase (SOD) and peroxidase are the principal enzymes that protect the male reproduction organs (Chatterjee et al., 2013). All studies that assessed the concentration of antioxidant enzymes, such as catalase, peroxidase, superoxide dismutase and glutathione in diabetic animals, reported decrease in their levels.

The methods employed were effective in recovering the levels of most antioxidant enzymes to control group 
levels. Nevertheless, Singh et al. (2013) found no significant increase in testicular glutathione S-transferase levels (GST) although Raza et al. (2004), Mallick et al. (2010), Shah and Khan (2014), Chatterjee et al. (2012, 2013) reported increase of GST levels in testis and/or sperm to the levels of control group. In studies by Mallick et al. (2007, 2010), Hamden et al. (2008), Giribabu et al. (2014) and Shah and Khan (2014), the treatment with plant extracts resulted in the recovery of catalase, SOD and glutathione peroxidase (GPx) levels.

Mallick et al. (2007) achieved the recovery of catalase and glutathione peroxidase levels and thus antioxidant protection to the testicular tissue. Hamden et al. (2008) noted that the levels of antioxidant enzymes were more efficiently recovered in animals treated with the extract prior to diabetes induction rather than those treated after induction. Further, there was an increase in enzyme cofactors levels, such as zinc, copper and iron, providing a protective effect against damage and death induced by the accumulation of free radicals. Reduction in enzyme cofactors levels is directly related to SOD decrease and to high blood glucose levels (Shah and Khan, 2014).

Chatterjee et al. (2012) noted that levels of catalase, peroxidase and superoxide dismutase increased after treatment, whereas Shah and Khan (2014) observed that extract administration enhanced the recovery of enzymes levels. Moreover, better results were achieved with higher extract doses. Ghosh et al. (2014) and Chatterjee et al. (2013) obtained the recovery of catalase, peroxidase and superoxide dismutase levels similar to the control group. In the study by Hajizadeh et al. (2014), the treatment recovered glutathione levels to higher rates than those of the control group. Moreover, Ukwenya et al. (2013) observed a recovery in SOD, with an increase in levels similar to control group and higher than insulin group.

Mallick et al. (2007), Chatterjee et al. (2012, 2013) and Ghosh et al. (2014) reported a decrease in oxidative markers that indicate the absence of metabolic toxicity of the used compounds. Thereby, medicinal plants comprise components, such as flavonoids and alkaloids, with hypoglycemic properties and antioxidant potential. They also play a fundamental role in the stimulation of endogenous production of protective enzymes and in the monitoring of the blood glucose levels in diabetic subjects (Mallick et al. 2010; Chatterjee et al., 2013; Ukwenya et al., 2013).

Further, $52.4 \%$ of studies measured the levels of testicular lipid peroxidation. In lipid peroxidation, the oxidizing substances, mostly ROS, attack the lipids with carbon-carbon double bond, especially polyunsaturated fatty acids in membranes, and cause apoptosis or necrosis (Yin et al., 2011; Ayala et al., 2014). Sperm plasma membrane has great amounts of the polyunsaturated fatty acid called docosahexaenoic acid, which makes it highly susceptible to oxidation (Suresh et al., 2013; Chatterjee et al., 2013; Giribabu et al., 2014). Increase in ROS levels, as observed in diabetic patients, changes membrane permeability, while the attack on mitochondrial membrane leads to reduced
ATP production and alteration of sperm motility and morphology (Amaral et al., 2006; Chatterjee et al., 2013).

The methods used by Mallick et al. (2010), Chatterjee et al. (2012, 2013), Suresh et al. (2013), Ukwenya et al. (2013) and Hajizadeh et al. (2014) were highly effective in reducing the high levels of lipid peroxidation in diabetic animals to the levels of control group. Mallick et al. (2007) and Singh et al. (2013) also achieved good results and Giribabu et al. (2014) and Shah and Khan (2014) observed that dose increase caused better recovery. Hamden et al. (2008) reported that preventive treatment before the onset of diabetes was more efficient for controlling lipid peroxidation levels than the treatment with diabetic animals.

Reactive oxygen species may also cause cell damage by the activation of apoptosis-inducing proteins, such as Bax (Chatterjee et al., 2012). Furthermore, induction of pro-apoptotic factors in germ cells is often related to testosterone concentration (Mallick et al., 2010). Thus, diabetic patients have an increase in apoptosis levels within the seminiferous tubules which may be linked to the reduction of steroidogenesis and increase in ROS production.

In $21 \%$ of the studies analyzed, apoptosis was evaluated within the seminiferous tubules through the identification of cell death in the germ cells and the expression of anti-apoptotic factors, such as Bcl-2, and pro-apoptotic factors, such as Bax.

In diabetic animals without treatment, Chatterjee et al. (2012, 2013) and Ghosh et al. (2014) observed increased rates of Bax gene, and Ghosh et al. (2014) noted the reduction of $\mathrm{Bcl}-2$ expression. However, in the three studies, treatment with plant extracts were efficient in the recovery of Bax levels and Bcl-2 expression to the levels of the control group. Mallick et al. (2010) also observed a reduction in the number of apoptotic germ cells in diabetic animals throughout the treatment. According to Mallick et al. (2010) and Chatterjee et al. (2012, 2013), reductions in cell death correspond to decrease in ROS peroxidation of the lipid membrane.

The control of apoptotic rates within the seminiferous tubules is important in regulating the population of germ cells and directly reflects sperm production (Clermont, 1972; Russell et al., 1990). The death of a large number of cells during the spermatogenic process may cause infertility due to cell loss (Ghosh et al., 2014). Besides the number of germ cells turning into spermatozoa, male fertility also depends on sperm motility and morphology (Venkatesh et al., 2009). In fact, 47.4, 31.6, 21 and 15.8\% of the studies respectively evaluated sperm production, sperm motility, sperm viability and sperm morphology.

All studies demonstrated that untreated diabetic rats had significant reductions in sperm number, motility and viability when compared to control group. Furthermore, Azeez et al. (2010) and Suresh et al. (2013) also observed alteration in sperm morphology. Treatments performed by Mallick et al. (2007, 2010), Chatterjee et al. (2012, 2013), Suresh et al. (2013) and Ghosh et al. (2014) resulted in some recovery of sperm parameters, whereas Giribabu et al. 
(2014) reported significant improvement with an increase in extract concentration. Hamden et al. (2008) had better results with preventive actions.

On the other hand, Azeez et al. (2010) did not report any improvement in sperm motility and morphology. Only the experimental group that received the highest extract dose $(1000 \mathrm{mg} / \mathrm{kg})$ showed recovery signs in sperm viability. The authors attributed the changes in sperm parameters of diabetic animals to oxidative damage. Since satisfactory results were not obtained, it seems that Cnidoscolus aconitifolius extract and the treatment used are not effective in controlling the production of oxidants.

In the study by Suresh et al. (2013), the Mucuna pruriens extract was effective in restoring the high number of spermatozoa with cytoplasmic droplets and protected the sperm DNA against damages in diabetic animals. These authors revealed that the plant species played an important role in suppressing ROS production and in stimulating antioxidant activity. Giribabu et al. (2014) observed a partial recovery in plasma membrane integrity and sperm morphology, directly related to the control of lipid peroxidation, hydrogen peroxide and nitric oxide production.

According to Chatterjee et al. (2013), the amount of sperm in diabetic animals decreases due to an increase in fructose concentration in the seminal plasma, as observed in $10.5 \%$ of studies. However, the methods used by Chatterjee et al. (2013) and Ghosh et al. (2014) reduced the semen fructose levels to levels of control animals.

Few studies investigated the characteristics of the seminiferous tubules: $21 \%$ observed tubular diameter and seminiferous epithelium integrity; $15.8 \%$ evaluated the number of germ cells at stage VII of the seminiferous epithelium cycle; $5.3 \%$ measured the seminiferous epithelium height. All authors observed injury in these parameters caused by diabetes. Mallick et al. (2007, 2010), Golalipour et al. (2011), Chatterjee et al. (2012) and Ghosh et al. (2014) slightly recovered the tubular diameter, epithelium height and number of germ cells at stage VII of the seminiferous epithelium cycle. The methods used by Golalipour et al. (2011), Chatterjee et al. (2012, 2013), Ukwenya et al. (2013) and Wankeu-Nya et al. (2013) partly restored the organization and integrity of the germinal epithelium.

Plasma testosterone level, performed in $42 \%$ of the assessed papers, was another parameter used for the evaluation of the testicular function. Untreated diabetic animals had a decrease in this hormone level in all studies. Such reductions have been attributed to the functionality of the Leydig cells. However, only Mallick et al. (2007) performed an analysis of the Leydig cells nuclear diameter and correlated it with decrease in testosterone levels.

Diabetes is associated with alterations in the hypothalamic-pituitary-testicular axis, resulting in significant reductions in the levels of hormones involved in spermatogenesis (Ballester et al., 2004; Agbaje et al., 2007). In addition, Leydig cells possess insulin receptors, or rather, they are dependent on optimal insulin concentrations for the efficient testosterone production (Brüning et al., 2000).
Mallick et al. (2007) reported a reduction in $\Delta 5.3 \beta$-hydroxysteroid dehydrogenase and $17 \beta$-hydroxysteroid dehydrogenase enzymatic activities, both involved in steroidogenesis, and a reduction in the nuclear area of Leydig cells. Decrease indicates the cells' functionality failure due to testosterone reduction in diabetic animals. Additionally, Chatterjee et al. (2012) observed an increase in cholesterol levels of diabetic animals, which impaired androgen production due to a lower usage of the cholesterol molecule. In Hamden et al. (2008), testicular total cholesterol levels were reduced in untreated diabetic animals. This may be due to the cholesterol glycation reactions or cholesterol-carriers with glucose, inhibiting the transportation to the testicle.

Despite the differences between the experimental results, the administration of plant extracts was effective in the rise of circulating testosterone levels, the restoration of the normal levels of cholesterol and the recovering of Leydig cell morphology.

All studies reported the increase of testosterone levels in treated groups. However, not all experiments succeeded in raising testosterone levels to the rates of the control group. The methods used by Mallick et al. (2010), Shahreari et al. (2010), Michael et al. (2013) and Ghosh et al. (2014) with plant extracts of Coccinia indica, Musa paradisiaca, Danae racemosa, Eugenia jambolana, and Phoenix dactylifera, respectively, proved to be highly efficient in recovering testosterone levels, with rates very close to and even higher than control group. Hamden et al. (2008) observed that the improvement of testosterone levels was more efficient in pre-diabetes induction group. Consequently, the extract had a protective effect, minimizing the reduction in testosterone levels. Nevertheless, the hormone levels were not as similar to the levels of the control group.

The amount of total protein was measured in $15.79 \%$ of the studies. All papers revealed reductions in protein levels in untreated diabetic animals. In a hyperglycemic state, glucose molecules may interact with proteins in non-enzymatic reactions to form advanced glycation end-products. High glycation leads to pathological complications that modify the functionality of various biological structures and results in the production of ROS (Barbosa et al., 2008). After treatment with plant extracts, the parameter was recovered. Shah and Khan (2014) observed an improvement directly related to the extract concentration, and the animals with the highest dose achieved results close to control group levels.

\section{Conclusion}

Results from all the studies under analysis showed that plant species have active principles in their composition that effectively help to control blood glucose levels and testicular oxidative damage triggered by diabetes. Although the antioxidant potential and the recoverability of reproductive parameters vary among species, these plants may be used as new alternatives to mitigate the effects of diabetes and improve the life quality of diabetic 
patients. Nevertheless, further analyses in testicular cells are required for a more complete conclusion on the benefits that the treatment provides to the organ's morphology and function. Additionally, toxicological analyses in organs, such as liver, kidneys and spleen, may be required to have a more precise picture of the extracts' applicability.

\section{References}

AGBAJE, I.M., ROGERS, D.A., MCVICAR, C.M., MCCLURE, N., ATKINSON, A.B., MALLIDIS, C. and LEWIS, S.E., 2007. Insulin dependant diabetes mellitus: implications for male reproductive function. Human Reproduction, vol. 22, no. 7, pp. 1871-1877. http://dx.doi.org/10.1093/humrep/dem077. PMid:17478459.

AMARAL, S., MORENO, A.J., SANTOS, M.S., SEICA, R. and RAMALHO-SANTOS, J., 2006. Effects of hyperglycemia on sperm and testicular cells of Goto-Kakizaki and streptozotocintreated rat models for diabetes. Theriogenology, vol. 66, no. 9, pp. 2056-2067. http://dx.doi.org/10.1016/j.theriogenology.2006.06.006. PMid:16860381.

AYALA, A., MUÑOZ, M.F. and ARGÜELLES, S., 2014. Lipid peroxidation: production, metabolism, and signaling mechanisms of malondialdehyde and 4-hydroxy-2-nonenal. Oxidative Medicine and Cellular Longevity, vol. 2014, pp. 360438. http://dx.doi. org/10.1155/2014/360438. PMid:24999379.

AZEEZ, O.I., OYAGBEMI, A.A., OYEYEMI, M.O. and ODETOLA, A.A., 2010. Ameliorative effects of Cnidoscolus aconitifolius on alloxan toxicity in Wistar rats. African Health Sciences, vol. 10, no. 3, pp. 283-291. PMid:21327141.

BALLESTER, J., MUÑOZ, M.C., DOMÍNGUEZ, J., RIGAU, T., GUINOVART, J.J. and RODRÍGUEZ-GIL, J.E., 2004. Insulin-dependent diabetes affects testicular function by FSH and LH-linked mechanisms. Journal of Andrology, vol. 25, no. 5, pp. 706-719. http://dx.doi.org/10.1002/j.1939-4640.2004. tb02845.x. PMid:15292100.

BARBOSA, J.H.P., OLIVEIRA, S.L. and SEARA, L.T., 2008. O papel dos produtos finais da glicação avançada (AGEs) no desencadeamento das complicações vasculares no diabetes. Arquivo Brasileiro de Endocrinologia Metabólica, vol. 52, no. 6, pp. 940950. http://dx.doi.org/10.1590/S0004-27302008000600005.</jrn>.

BRÜNING, J.C., GAUTAM, D., BURKS, D.J., GILLETTE, J., SCHUBERT, M., ORBAN, P.C., KLEIN, R., KRONE, W., MÜLLER-WIELAND, D. and KAHN, C.R., 2000. Role of brain insulin receptor in control of body weight and reproduction. Science, vol. 289 , no. 5487, pp. 2122-2125. http://dx.doi.org/10.1126/ science.289.5487.2122. PMid:11000114.

CANSIAN, R.L., KUBIAK, G.B., BORSATTI, L., MIELNICZKIPEREIRA, A.A., ROMAN, S.S., PAROUL, N., SCHWANZ, M., MANFREDINI, V. and MOSSI, A.J., 2015. Antioxidant and genotoxic properties of Maytenus dasyclada: a comparative study in relation to Maytenus reference species. Brazilian Journal of Biology $=$ Revista Brasileira de Biologia, vol. 75, no. 2, pp. 471476. http://dx.doi.org/10.1590/1519-6984.18113. PMid:26132034.

CHATTERJEE, K., ALI, K.M., DE, D., BERA, T.K., JANA, K., MAITI, S., GHOSH, A., SAMANTA, R. and GHOSH, D., 2012. Diabetes induced testicular dysfunction amelioration by ethyl acetate fraction of hydromethanolic extract of root of Musa paradisiaca L. in streptozotocin induced diabetic rat. Asian Pacific Journal of Tropical Disease, vol. 2, no. 1, pp. 233-241. http:// dx.doi.org/10.1016/S2222-1808(12)60158-3.
CHATTERJEE, K., ALI, K.M., DE, D., BERA, T.K., JANA, K., MAITI, S., GHOSH, A. and GHOSH, D., 2013. Hyperglycemiainduced alteration in reproductive profile and its amelioration by the polyherbal formulation MTEC (modified) in streptozotocininduced diabetic albino rats. Biomarkers and Genomic Medicine, vol. 5, no. 1-2, pp. 54-66. http://dx.doi.org/10.1016/j.gmbhs.2013.04.007.

CLERMONT, Y., 1972. Kinetics of spermatogenesis in mammals: seminiferous epithelium cycle and spermatogonial renewal. Physiological Reviews, vol. 52, no. 1, pp. 198-236. PMid:4621362.

DOWLING, D.K. and SIMMONS, L.W., 2009. Reactive oxygen species as universal constraints in life-history evolution. Proceedings of Royal Society Part B, Biological Sciences, vol. 276, no. 1663, pp. 1737-1745. http://dx.doi.org/10.1098/rspb.2008.1791. PMid:19324792.

FRANÇA, L.R. and RUSSELL, L.D., 1998. The testis of domestic mammals. In: F. MARTÍNEZ-GARCIA and J. REGADERA. Male reproduction: a multidisciplinary overview. Madrid: Churchill Communications, pp. 198-219.

GHOSH, A., JANA, K., ALI, K.M., DE, D., CHATTERJEE, K. and GHOSH, D., 2014. Corrective role of Eugenia jambolana on testicular impairment in streptozotocin-induced diabetic male albino rat: An approach through genomic and proteomic study. Andrologia, vol. 46, no. 3, pp. 296-307. http://dx.doi. org/10.1111/and.12081.

GHOSH, S. and ROY, T., 2013. Evaluation of antidiabetic potential of methanolic extract of Coccinia indica leaves in streptozotocin induced diabetic rats. International Journal of Pharmaceutical Sciences and Research, vol. 4, no. 11, pp. 4325-4328.

GIRIBABU, N., KUMAR, K.E., REKHA, S.S., MUNIANDY, S. and SALLEH, N., 2014. Chlorophytum boviriliarum (Safed Musli) root extract prevents impairment in characteristics and elevation of oxidative stress in sperm of streptozotocin induced adult male diabetic rats. BMC Complementary and Alternative Medicine, vol. 14, no. 291, pp. 377. PMid:25283233.

GOLALIPOUR, M.J., KABIRI BALAJADEH, B., GHAFARI, S., AZARHOSH, R. and KHORI, V., 2011. Protective effect of Urtica dioica L. (Urticaceae) on morphometric and morphologic alterations of seminiferous tubules in STZ diabetic rats. Iranian Journal of Basic Medical Sciences., vol. 14, no. 5, pp. 472-477. PMid:23493848.

HAJIZADEH, M., EFTEKHAR, E., ZAL, F., JAFARIAN, A. and MOSTAFAVI-POUR, Z., 2014. Mulberry leaf extract attenuates oxidative stress mediated testosterone depletion in streptozotocin induced diabetic rats. Iranian Journal of Medical Sciences, vol. 39, no. 2, pp. 123-129. PMid:24644381.

HAMDEN, K., CARREAU, S., JAMOUSSI, K., AYADI, F., GARMAZI, F., MEZGENNI, N. and ELFEKI, A., 2008. Inhibitory effects of 1alpha, 25dihydroxyvitamin D3 and Ajuga iva extract on oxidative stress, toxicity and hypofertility in diabetic rat testes. Journal of Physiology and Biochemistry, vol. 64, no. 3, pp. 231239. http://dx.doi.org/10.1007/BF03216108. PMid:19244937.

MALLICK, C., MANDAL, S., BARIK, B., BHATTACHARYA, A. and GHOSH, D., 2007. Protection of testicular dysfunctions by MTEC, a formulated herbal drug, in streptozotocin induced diabetic rat. Biological \& Pharmaceutical Bulletin, vol. 30, no. 1, pp. 84-90. http://dx.doi.org/10.1248/bpb.30.84. PMid:17202665.

MALLICK, C., BERA, T.K., ALI, K.M., CHATTERJEE, K. and GHOSH, D., 2010. Diabetes-induced testicular disorders vis-avis germ cell apoptosis in albino rat: Remedial effect of hexane fraction of root of Musa paradisiaca and leaf of Coccinia indica. 
Journal of Health Science, vol. 56, no. 6, pp. 641-654. http:// dx.doi.org/10.1248/jhs.56.641.

MARITIM, A., SANDERS, R. and WATKINS 3RD, J., 2003. Diabetes, oxidative stress, and antioxidants: a review. Journal of Biochemical and Molecular Toxicology, vol. 17, no. 1, pp. 24-38. http://dx.doi.org/10.1002/jbt.10058. PMid:12616644.

MICHAEL, H.N., SALIB, J.Y. and ESKANDER, E.F., 2013. Bioactivity of diosmetin glycosides isolated from the epicarp of date fruits, Phoenix dactylifera, on the biochemical profile of alloxan diabetic male rats. Phytotherapy Research, vol. 27, no. 5, pp. 699-704. http://dx.doi.org/10.1002/ptr.4777. PMid:22761049.

MODAK, M., DIXIT, P., LONDHE, J., GHASKADBI, S. and DEVASAGAYAM, T.P., 2007. Indian herbs and herbal drugs used for the treatment of diabetes. Journal of Clinical Biochemistry and Nutrition, vol. 40, no. 3, pp. 163-173. http://dx.doi.org/10.3164/ jcbn.40.163. PMid:18398493.

RATNER, R.E., HIRSCH, I.B., NEIFING, J.L., GARG, S.K., MECCA, T.E. and WILSON, C.A., 2000. Less hypoglycemia with insulin glargine in intensive insulin therapy for type 1 diabetes. Diabetes Care, vol. 23, no. 5, pp. 639-643. http://dx.doi. org/10.2337/diacare.23.5.639. PMid:10834423.

RAZA, H., AHMED, I. and JOHN, A., 2004. Tissue specific expression and immunohistochemical localization of glutathione S-transferase in streptozotocin induced diabetic rats: modulation by Momordica charantia (karela) extract. Life Sciences, vol. 74, no. 12, pp. 1503-1511. http://dx.doi.org/10.1016/j.lfs.2003.08.023. PMid:14729399.

RUSSELL, L.D., ETTLIN, R.A., SINHA HIKIM, A.P. and CLEGG, E.D., 1990. Histological and histopathological evaluation of the testis. Clearwater: Cache River Press. 286 p.

SALIB, J.Y., MICHAEL, H.N. and ESKANDE, E.F., 2013. Antidiabetic propertiers of flavonoid compounds isolated from Hyphaene thebaica epicarpo on alloxan induced diabetic rats. Pharmacognosy Research, vol. 5, no. 1, pp. 22-29. http://dx.doi. org/10.4103/0974-8490.105644. PMid:23598921.

SHAH, N.A. and KHAN, M.R., 2014. Antidiabetic effect of Sida cordata in alloxan induced diabetic rats. BioMed Research International, vol. 2014, pp. 671294. http://dx.doi. org/10.1155/2014/671294. PMid:25114914.

SHAHREARI, S., KHAKI, A., AHMADI-ASHTIANI, H.R., REZAZADEH, S. and HAJIAGHAEI, R., 2010. Effects of Danae racemosa on testosterone hormone in experimental diabetic rats. Journal of Medicinal Plants, vol. 9, no. 35, pp. 114-119.

SINGH, R., BHARDWAJ, P. and SHARMA, P., 2013. Antioxidant and toxicological evaluation of Cassia sopherain streptozotocininduced diabetic Wistar rats. Pharmacognosy Research, vol. 5, no. 4, pp. 225-232. http://dx.doi.org/10.4103/0974-8490.118767. PMid:24174814.

SURESH, S., PRITHIVIRAJ, E., LAKSHMI, N.V., GANESH, M.K., GANESH, L. and PRAKASH, S., 2013. Effect of Mucuna pruriens (Linn.) on mitochondrial dysfunction and DNA damage in epididymal sperm of streptozotocin induced diabetic rat. Journal of Ethnopharmacology, vol. 145, no. 1, pp. 32-41. http://dx.doi. org/10.1016/j.jep.2012.10.030. PMid:23103904.

TAKAO, L.K., IMATOMI, M. and GUALTIERI, S.C., 2015. Antioxidant activity and phenolic content of leaf infusions of Myrtaceae species from Cerrado (Brazilian Savanna). Brazilian Journal of Biology $=$ Revista Brasileira de Biologia, vol. 75, no. 4, pp. 948-952. http://dx.doi.org/10.1590/1519-6984.03314. PMid:26675912.

UKWENYA, V., ASHAOLU, O., ADEYEMI, D., OBUOTOR, E., TIJANI, A., BILIAMINU, A. and CAXTON-MARTINS, E., 2013. Evaluation of antioxidant potential of methanolic leaf extract of Anacardium occidentale on the testes of streptozotocin induced diabetic wistar rats. European Journal of Anatomy, vol. 2 , no. 17 , pp. $72-81$.

VAN BELLE, T., COPPIETERS, K. and VON HERRATH, M., 2011. Type1 diabetes: etiology, immunology, and therapeutic strategies. Physiological Reviews, vol. 91, no. 1, pp. 79-118. http://dx.doi.org/10.1152/physrev.00003.2010. PMid:21248163.

VENKATESH, S., SINGH, G., GUPTA, N.P., KUMAR, R., DEECARAMAN, M. and DADA, R., 2009. Correlation of sperm morphology and oxidative stress in infertile men. Iranian Journal of Reproductive Medicine, vol. 7, no. 1, pp. 29-34.

WANKEU-NYA, M., FLOREA, A., BÂLICI, S., WATCHO, P., MATEI, H. and KAMANYI, A., 2013. Dracaena arborea alleviates ultra-structural spermatogenesis alterations in streptozotocin induced diabetic rats. BMC Complementary and Alternative Medicine, vol. 13, pp. 71. PMid:23548080.

YIN, H., XU, L. and PORTER, N.A., 2011. Free radical lipid peroxidation: mechanisms and analysis. Chemical Reviews, vol. 111, no. 10, pp. 5944-5972. http://dx.doi.org/10.1021/cr200084z. PMid:21861450. 